Original article

\title{
Measurement of negative and depressive symptoms: Discriminatory relevance of affect and expression
}

\author{
Janina Richter*, Lea Hölz, Klaus Hesse, Dirk Wildgruber, Stefan Klingberg \\ Department of Psychiatry and Psychotherapy, University of Tuebingen, Calwerstrasse 14, 72076 Tuebingen, Germany
}

\section{A R T I C L E I N F O}

\section{Article history:}

Received 13 July 2018

Received in revised form 29 August 2018

Accepted 28 September 2018

Available online 29 October 2018

\section{Keywords:}

Schizophrenia

Depression

Negative symptoms

Psychometry and assessments in psychiatry

\begin{abstract}
A B S T R A C T
There is conceptual overlap between negative and depressive symptoms: Mainly the 'avolition' factor of negative symptoms also encompasses main symptoms of depression. However, whereas in depression mood is low, mainly anticipatory anhedonia can be found in negative symptoms. Moreover, patients with schizophrenia (SCZ) show greater expressive deficits than those with Major Depressive Episode (MDE). We investigated if measures of depressive and negative symptoms differentiate SCZ subjects, subjects with MDE, and healthy controls (HC). 21 SCZ, $22 \mathrm{MDE}$, and $25 \mathrm{HC}$ subjects were examined with a rater assessment and a self-rating for negative symptoms (Clinical Assessment Interview for Negative Symptoms (CAINS); Motivation and Pleasure - Self-Report (MAP-SR)) and depressive symptoms (Hamilton Rating Scale for Depression (HAMD-17); Beck Depression Inventory (BDI)). All measures differentiated the psychiatric samples from HC(all p's < 0.01). The ratings of depressive symptoms (HAMD-17, BDI) and rater assessment of negative symptoms (CAINS) - specifically its sub scale measuring expressive deficits - managed to discriminate between subjects with schizophrenia and those with MDE (SCZ > MDE > HC for negative, $\mathrm{MDE}>\mathrm{SCZ}>\mathrm{HC}$ for depressive symptoms, all p's $<0.05$ ). The self-rating of negative symptoms (MAP-SR) did not. To differentiate negative symptoms and depression clinicians might look for (self-)reported low mood and observer-rated reduction in speech as well as in gestures and facial expression. Reduced expression and moderate levels of depression point towards a negative syndrome, whereas mostly unimpaired expression and high scores of self-reported depressive symptoms are more likely to indicate a depressive syndrome.
\end{abstract}

(c) 2018 Elsevier Masson SAS. All rights reserved.

\section{Introduction}

\subsection{Schizophrenia and depression}

There is sound evidence pointing to a two-factorial structure of negative symptoms; the first factor being "diminished expression" with blunted expression of emotions and poverty of speech and the second being "avolition" with amotivation, anhedonia and social withdrawal [e.g. 1]. Secondary negative symptoms are caused by positive symptoms, substance use, medication side effects and/or particularly important here - depression [e.g. 2]. Negative and depressive symptoms can't be differentiated easily, since there is considerable conceptual overlap. Mainly the avolition factor of negative symptoms encompasses symptoms that also belong to

\footnotetext{
* Corresponding author.

E-mail addresses: janina.richter@med.uni-tuebingen.de, janinarichter@gmx.de (J. Richter), lea.hoelz@med.uni-tuebingen.de (L. Hölz), klaus.hesse@med.uni-tuebingen.de (K. Hesse), dirk.wildgruber@med.uni-tuebingen.de (D. Wildgruber), stefan.klingberg@med.uni-tuebingen.de (S. Klingberg).
}

the main symptoms of depression: loss of interest, anhedonia, and reduced energy.

Comorbidity rates are high for schizophrenia and unipolar depression across stage and state of illness (acute psychotic episode: up to $60 \%$ comorbid major depressive episode (MDE); post-psychotic: moderate to severe MDE in $20 \%$ of chronic patients and $50 \%$ of first-episode patients); there seem to be shared aetiological aspects [3]. Longitudinally, up to $80 \%$ of patients with schizophrenia experience an episode of major depression [4]. Depression is the most important indicator for completed suicide in patients with schizophrenia [5]. Since both the treatment of depression in schizophrenia and of negative symptoms remains inadequate [e.g. 6,7], it seems of particular importance to reliably delineate negative and depressive symptoms.

\subsection{Expression, mood and subtypes of anhedonia might differentiate schizophrenia and depression}

Emotional expression (i.e. speech, gestures and facial expressions) often is reduced in patients with schizophrenia compared to healthy controls and subjects with depression, which are nevertheless also showing expressional deficits [8-11]. Despite 
affective flattening, patients with schizophrenia often report unimpaired subjective experiences [e.g. 12], whereas low, depressed mood is a main symptom of depression. Recent conceptualizations of anhedonia emphasize the importance of the (complex) reward system. Any reward system deficit (e.g. anticipatory or motivational anhedonia, disorganization) can hinder the individual from generating pleasurable experiences and could then present as (secondary) consummatory anhedonia [13]. There are findings indicating that patients with depression experience consummatory and anticipatory anhedonia whereas patients with schizophrenia mainly show a deficit in anticipatory pleasure [13-15]. The emergence of anticipatory pleasure is more complex than the experience of consummatory pleasure and hints at motivational deficits in schizophrenia as opposed to deficits in experiencing emotions [16].

\subsection{Correlations of rating scales for depression and negative symptoms}

Research on negative symptoms in the context of MDE seems scarce. Bottlender, Sato [17] found that negative symptoms (measured with the Scale for the Assessment of Negative Symptoms (SANS)) were significantly associated with depressive symptoms (measured with the Montgomery-Asberg Depression Rating Scale) in MDE patients but not in patients with schizophrenia. This could be due to the SANS' item content that encompasses a lot of symptoms also germane to the depressive domain (e.g. affective nonresponsivity, poverty of content of speech, increased latency of response) and symptoms that are not thought to be specific for negative symptoms anymore, i.e. attention/cognitive symptoms [e.g. 1]. They found persisting negative symptoms to be indicative for schizophrenia and not MDE.

For schizophrenia patients, Park, Llerena [18] found a weak correlation between observer-rated negative symptoms (CAINS) and the rater assessed Calgary Depression Scale for Schizophrenia (CDSS), Kring, Gur [19] found none. Engel, Fritzsche [20] reported no significant association of CAINS and self-assessed BDI-II. Llerena, Park [21] found no significant correlation of self-rated negative symptoms (MAP-SR) with the CDSS. Hartmann, Fritzsche [22] reported no significant correlation between the BDI-II and PANSS-rated negative symptoms (however, it should be noted that two of the seven PANSS negative items assesses cognitive symptoms [e.g. 1]). Engel and Lincoln [23] reported a moderate and significant correlation of the MAP-SR with the BDI-II $(r=0.39)$. Overall, we found some - if scarce - evidence for overlap when measuring the two symptom domains in patients with schizophrenia. Concerning self- vs. observer-ratings, Engel and Lincoln [23] debated an underestimation of shared variance of negative and depressive symptoms when compared across sampling methods.

\subsection{Objectives}

We investigated if a) measures of negative symptoms and b) measures of depressive symptoms could differentiate between subjects with MDE, subjects with schizophrenia and healthy controls. We expect subjects with schizophrenia to show the greatest extent of negative symptoms and subjects with depression to show the greatest extent of depressive symptoms. Because of the overlap between negative symptoms and depression we expect subjects with schizophrenia to display more depressive symptoms and subjects with MDE to report more negative symptoms than healthy controls. We expect mainly the "expression" factor of negative symptoms and the assessments of depressive mood to reliably differentiate MDE and schizophrenia subjects.

\subsection{Selection of instruments}

To assess the scope of negative symptoms we used the Clinical Assessment Interview for Negative Symptoms (CAINS, Engel, Fritzsche [20]) and the self-rating instrument Motivation and Pleasure Scale - Self-Report (MAP-SR, Engel and Lincoln [23]). The CAINS has been designed to assess negative symptoms according to the current conceptualization [1] and consists of two scales: "motivation and pleasure" (CAINS-MAP) and "expression" (CAINSEXP). CAINS-EXP straightforwardly rater-assesses expressive deficits with four items. CAINS-MAP focuses on aspects of inner experience with its authors arguing that this is central for the emotional, social and motivational deficits and to be distinguished from behavior or functional outcome [19]. In the original validation study, the two factors correlate moderately $(r=0.24)$, show good internal consistency as well as test-retest reliability and interrater reliability. Convergent and discriminant validity (also to depressive symptoms) was established [19]. A further validation study found good psychometric properties for the German CAINS as well, with high internal consistency, a moderate correlation between the two factors $(r=0.44)$, good inter-rater agreement as well as convergent and discriminant validity; the latter also with depression [20]. The MAP-SR assesses the "avolition" factor of negative symptoms as a self-report and is based on the CAINS' "motivation and pleasure" scale. It taps social pleasure, recreational or work pleasure, feelings and motivations about close, caring relationships as well as motivation and effort to engage in activities. Llerena, Park [21] found good internal consistency as well as convergent validity with the CAINS-MAP $(r=0.65)$ and social anhedonia $(r=0.48)$. For social performance, there was no significant correlation. Discriminant validity was established (i.a. for depression/anxiety). For the German MAP-SR, Engel and Lincoln [23] also found high internal consistency as well as mostly good convergent and discriminant validity. However, there was a moderate correlation with the BDI-II $(\mathrm{r}=0.39)$.

To assess the scope of depressive symptoms, we used the Beck Depression Inventory (BDI, Hautzinger [24]) as self-rating and the Hamilton Depression Scale (HAMD-17, Hamilton [25]) as rater assessment. Both tap emotional, cognitive, behavioral, and physical symptoms of depression. The BDI consists of 17 items and shows good validity, adequate test-retest reliability, and good inner consistency [26]. For the German BDI high internal consistency and good convergent validity is reported [24]. Concerning the observer rating, we expect greater discriminatory power from the HAMD's 17-item version as opposed to HAMD-21, since this version doesn't assess paranoia and depersonalization. The HAMD-17 is widely used and has good to adequate psychometric properties [e.g. 27]. Because we expect items associated with mood to have the most discriminatory power, we researched established subscales with emphasis on mood for BDI and HAMD-17, respectively. The BDI subscale "cognitive/ affective" consists of the first 14 items excluding somatic and functioning items [26]. The Maier-Philipp Severity subscale of the HAMD-17 comprises the items assessing depressed mood, feelings of guilt, work and interests, retardation, agitation, and anxiety psychic [28].

\section{Methods}

\subsection{Participants}

We included 21 participants with schizophrenia (SCZ), 22 participants with MDE and 25 healthy controls (HC). The patients were recruited from in-patient settings. Inclusion criteria were diagnosis of a psychotic disorder (SCZ) or major depressive episode (MDE) or no diagnosis (HC) according to DSM-IV (assessed with the 
German Brief Diagnostic Interview of Mental Disorders (MiniDIPS)), age 18 to 65 years, sufficient German language skills, normal or corrected to normal vision and hearing as well as capability to give consent. To avoid overlap between the psychiatric groups we did not include subjects with schizoaffective disorder or a MDE with psychotic symptoms. Since we wanted to measure present symptoms, we excluded patients in remission (i.e. only met "life time" criteria in the diagnostic interview). Further exclusion criteria were substance dependence as leading clinical problem and intellectual disability. To keep the sample structure comparable, we included healthy controls that corresponded in age and gender to the recruited SCZ patients. Since depressed patients skew older and female and we prioritized a representative sample, we refrained from doing that with the MDE sample. Demographic and clinical characteristics of the samples can be found in Table 1.

\subsection{Measures and procedure}

The study protocol was approved by the ethics committee of the University of Tuebingen's medical faculty. After obtaining informed consent, screened participants who met inclusion criteria were interviewed and asked to fill in questionnaires (duration approx. $1.5 \mathrm{~h}$ ). Diagnosis was confirmed using the parts of the German Brief Diagnostic Interview of Mental Disorders (MiniDIPS) that assess psychotic and affective disorders.

The German translation of the CAINS was kindly made available to us by the research group led by Tania Lincoln, Department of Clinical Psychology and Psychotherapy, University of Hamburg. The English version of the MAP-SR was translated into German by our research group and retranslated by an English native speaker. Differences to the original English versions were discussed among the translators and a consensus was agreed on.

\subsection{Data analysis}

For demographic data a rate of missings $<10 \%$ was not reported. When calculating the self-ratings' scale composites, a rate of $10 \%$ of missings was tolerated and replaced by the scale's mean. Measures with more missing data were excluded from the analysis (one data point for MAP-SR and BDI, respectively). There were no missing data for the rater assessments. Scores for the MAP-SR were inverted so that larger scores indicate a greater extent of negative symptoms. With SPSS 25.0 we tested for normal distribution with the Shapiro-Wilk test. Since CAINS, MAP-SR, HAMD-17 and BDI and their subscales were all non-normally distributed in the control group, we used the non-parametrical Kruskal-Wallis-Test to assess the between-group effects. Post-hoc pairwise comparisons were performed using Dunn tests with Bonferroni correction.

\section{Results}

For the CAINS we found between-group differences in the scope of negative symptoms $(H(2, N=68)=48.65, p<.001)$. Post-hoc analysis revealed significant differences between all groups: $\mathrm{SCZ}>$

Table 1

Sample demographics.

\begin{tabular}{llll}
\hline Demographics & & & \\
& $\mathrm{SCZ}(\mathrm{n}=21)$ & $\mathrm{MDE}(\mathrm{n}=22)$ & $\mathrm{HC}(\mathrm{n}=25)$ \\
\hline Age (yrs) & $35.10(11.68)$ & $42.36(15.05)$ & $34.28(14.24)$ \\
Male (\%) & 86 & 50 & 68 \\
Abitur ( A-level; \%) & 81 & 54 & 88 \\
Age at $1^{\text {st }}$ hospitalization (yrs) & $23.71(4.37)$ & $35.36(13.11)$ & - \\
\hline
\end{tabular}

Notes. SCZ = participants with schizophrenia; $\mathrm{MDE}=$ participants with major depressive disorders; $\mathrm{HC}=$ healthy controls.
MDE $>$ HC, $p<0.05$. MAP-SR also revealed differences between the groups $(H(2, \mathrm{~N}=68)=25.77, p<.001)$. Here, post-hoc analysis showed SCZ $=$ MDE $>$ HC with $p<.001$ for MDE and controls and $p=.007$ for SCZ and controls. Because of the MAP-SR's failure to differentiate SCZ and MDE, we further analyzed the CAINS' two subscales and found that only its expression subscale significantly differentiated between subjects with depression and schizophrenia (SZC > MDE > HC). The group comparisons of the negative symptom scales can be found in Fig. 1.

Concerning the scope of depressive symptoms we found between-group differences for $\operatorname{BDI}(H(2, \mathrm{~N}=67)=41.83, p<$ 0.001 ). Post-hoc analysis showed that all three groups differed significantly from each other: $\mathrm{MDE}>\mathrm{SCZ}>\mathrm{HC}, p<0.05$. For HAMD-17, there were significant differences as well $(H(2$, $\mathrm{N}=68)=54.14, p<0.001)$. Post-hoc tests showed MDE $>\mathrm{SCZ}>$ HC, $p<0.05$; see Fig. 2 .

For the "mood associated" subscales of the measures for depressive symptoms, we found both to not significantly differentiate between depressed and schizophrenic subjects, while still showing between-group differences. HAMD-Maier-Philipp: $H$ $(2, \mathrm{~N}=68)=53.32, p<0.001, \mathrm{MDE}=\mathrm{SCZ}>\mathrm{HC}$; BDI cognitive/ affective: $H(2, \mathrm{~N}=67)=37.12, p<0.001, \mathrm{MDE}=\mathrm{SCZ}>\mathrm{HC}$. The "other" subscale of the HAMD17 as well as the somatic subscale of the BDI differentiated significantly between all groups. HAMD other: $H(2, \mathrm{~N}=68)=43.74, p<.001, \mathrm{SZC}>\mathrm{MDE}>\mathrm{HC}$; BDI somatic $\mathrm{H}$ $(2, \mathrm{~N}=67)=40.11, \mathrm{p}<.001, \mathrm{MDE}>\mathrm{SCZ}>\mathrm{HC}$. The group comparisons of the depression measures' subscales can be found in Fig. 3 .

\section{Discussion}

We investigated if subjects with schizophrenia, subjects with MDE, and healthy controls differ in the scope of their negative and depressive symptoms as measured by self-ratings (BDI, MAP-SR) and observer assessments (HAMD-17, CAINS). All measures differentiated the psychiatric samples from the controls. The full
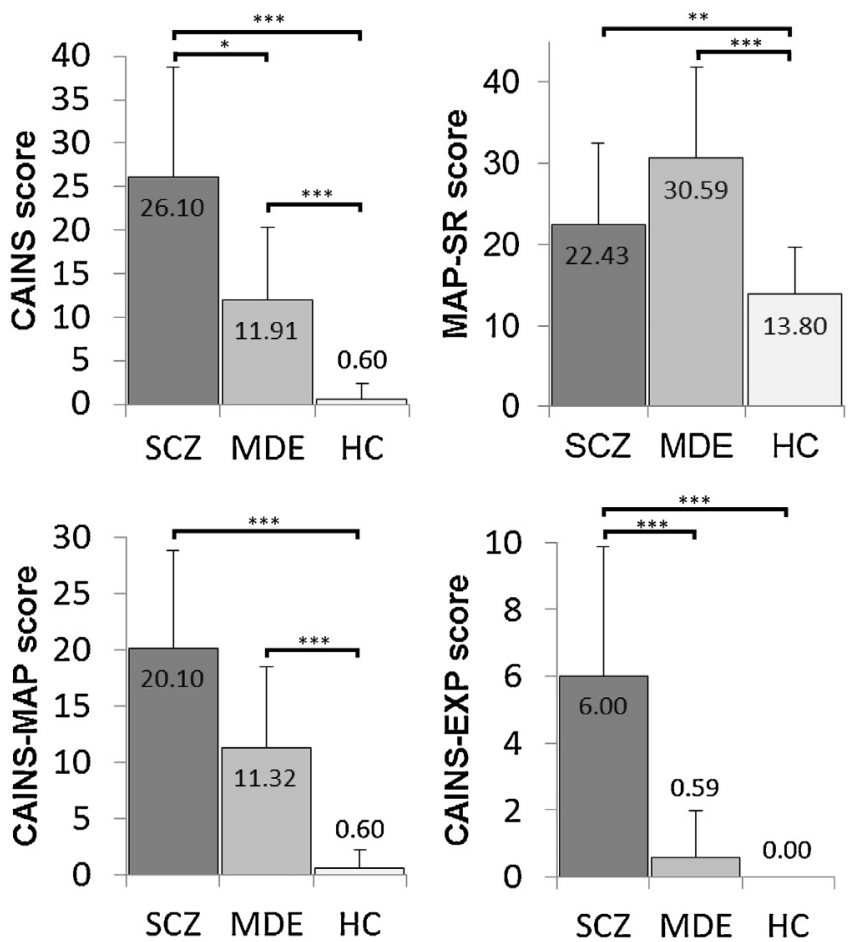

Fig. 1. Group comparisons of CAINS and MAP-SR, and CAINS-MAP and CAINS-EXP; means with standard deviations and significance markers. 

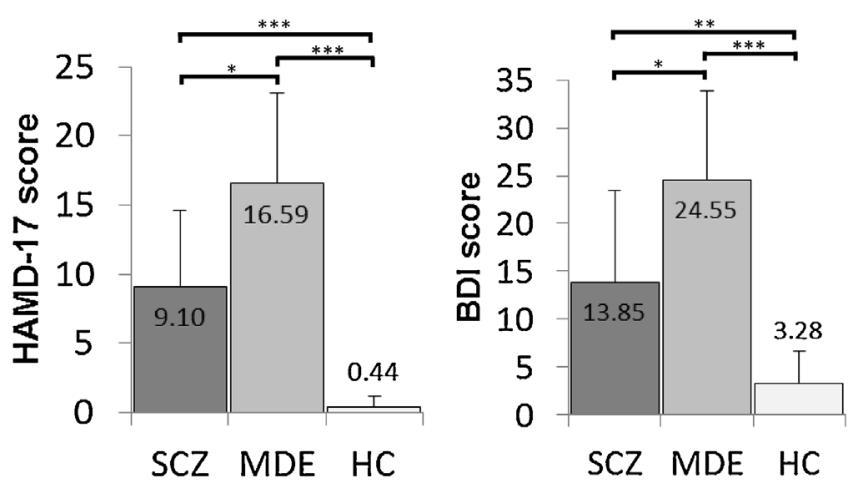

Fig. 2. Group comparisons of HAMD-17 and BDI; means with standard deviations and significance markers.

Note. ${ }^{*}=p<0.05,{ }^{* *}=p<0.01,{ }^{* * *}=p<0.001$.
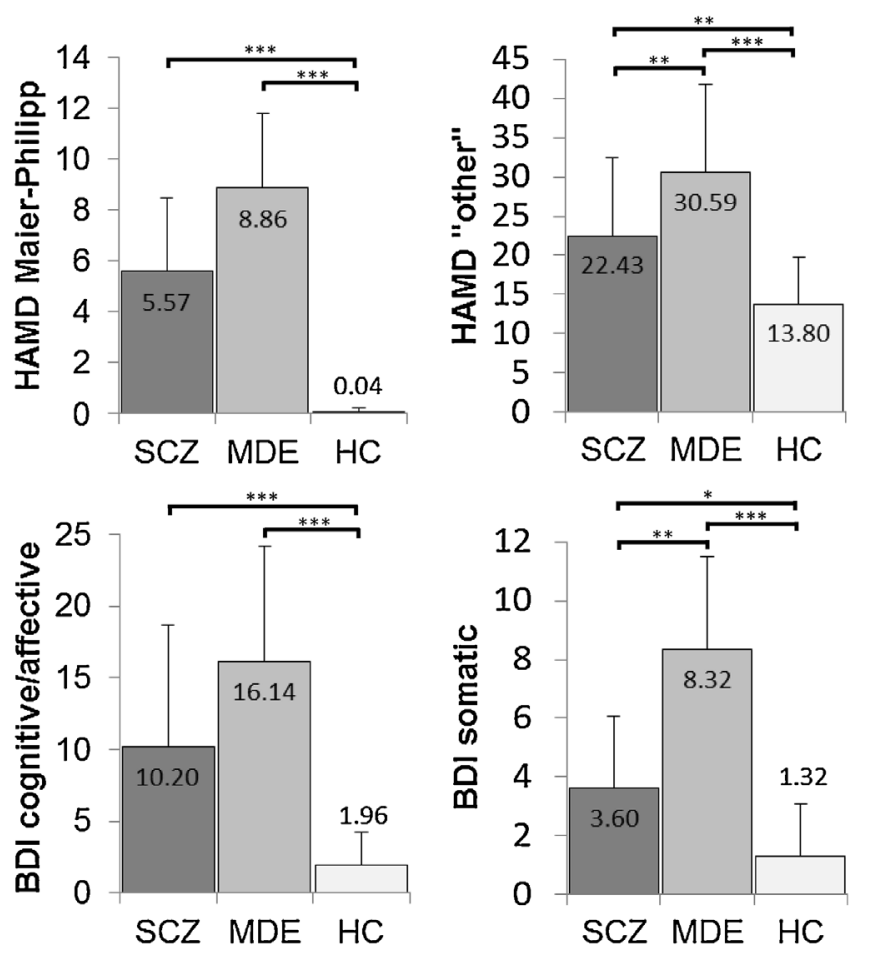

Fig. 3. Group comparisons of HAMD-17 Maier-Philipp and "other" subscales, and BDI cognitive/affective and somatic subscales; means with standard deviations and significance markers.

Note. ${ }^{*}=p<0.05,{ }^{* *}=p<0.01,{ }^{* * *}=p<0.001$.

rating scales of depressive symptoms (HAMD-17, BDI) and the rater assessment of negative symptoms (CAINS) - and specifically the expressive deficits (CAINS-EXP) - managed to discriminate between subjects with schizophrenia and those with MDE reliably; the self-rating of negative symptoms (MAP-SR) did not.

Concerning the CAINS, its "expression" subscale (CAINS-EXP, assessing vocal prosody, gestures, facial expression, and speech) significantly differentiates schizophrenic and depressed subjects whereas the "avolition" subscale does not (although its means also are $\mathrm{SCZ}>\mathrm{MDE}>\mathrm{HC}$ ). This differentiating effect of the CAINS-EXP is consistent with previous evidence that shows reduced facial expression of subjects with schizophrenia compared to subjects with depression (which are nevertheless also showing diminished expression); this also holds true for involuntary facial activity $[8,9,29]$. Gaebel and Wolwer [10] found diminished expression beyond acute psychotic episodes in schizophrenia patients; in subjects with depression this was primarily found when acutely depressed. One explanation for the MAP-SR's failure to distinguish the psychiatric groups could be poor self-assessment by subjects with schizophrenia. There is evidence for deficits in self-assessment, with mainly positive symptoms and cognitive symptoms negatively affecting the ability to self-assess correctly [30-32]. However, Hartmann, Fritzsche [22] assessed patients with psychosis for depression with two observer ratings (CDSS and PANSS) and two self-rating scales (BDI and Symptom-Checklist Revised (SCL-90-R) and found self-ratings to correspond well with observer ratings. Since the CAINS "expression" subscale seems to play the decisive role, the MAP-SR's failure to differentiate the psychiatric samples could be because it doesn't measure expression. Moreover, in contrast to the means of the CAINS "motivation and pleasure" sub-scale (SCZ > MDE $>\mathrm{HC}$ ), MDE-subjects reported greater symptom load on the MAP-SR than subjects with schizophrenia (MDE $>\mathrm{SCZ}>\mathrm{HC}$ ). The MAP-SR doesn't seem to measure symptoms in the "motivation and pleasure" domain that are specific for anhedonia/avolition in schizophrenia. To differentiate between depressive symptoms and negative symptoms both instruments might not assess enough items that allow for a discrimination of subtypes of anhedonia (anticipatory vs. consummatory anhedonia) and mood symptoms (indifferent vs. depressed mood). Therefore, future research on instruments assessing the "amotivation" factor of negative symptoms should focus on a more detailed assessment of these symptom domains, if the aim is to differentiate reliably from depression. In conclusion, the findings of the current study indicate that the "expression" factor of negative symptoms as assessed with the CAINS-EXP can differentiate MDE and negative symptoms of schizophrenia, whereas both scales measuring the "amotivation" factor (CAINS-MAP and MAP-SR) do not allow for a specific distinction.

In this study, we used the BDI; the newer BDI-II additionally inquires agitation, worthlessness, loss of energy, and concentration difficulty and dropped body image change, work difficulty, weight loss, and somatic preoccupation. Interestingly, Hartmann, Fritzsche [22] found that schizophrenia patients who selfreported fewer depressive symptoms than the clinicians observed, showed more negative symptoms, i.e. blunted affect and poor affective rapport. We also found slightly greater mean differences between schizophrenia patients and to MDE subjects in the self-assessment of depressive symptoms than in the observer rating. We supposed that while the HAMD-17 manages to differentiate patients with MDE and those with SCZ, there are some items that might reduce discriminatory power: four inquire physical symptoms, and three sleep problems. Patients with schizophrenia as well as clinically depressed patients both experience and report more physical symptoms [e.g. 33, 34]. Particularly sexual dysfunction is a common side effect of antipsychotics [e.g. 35]. Moreover, 30 to $80 \%$ of schizophrenic patients also suffer from sleep disturbances [36]. Curiously, we could confirm this neither when analyzing the subscales of the HAMD-17 nor of the BDI. Focus on psychic symptoms and affective and cognitive symptoms of depression, respectively, did not increase discriminatory power. Conversely, the scales' items that feature somatic and sleep symptoms seem to differentiate the psychiatric samples better. Maybe the subscales were still not specific enough for affective/mood symptoms. This warrants further research on the differential impact of the scales' singular items. At this point, however, we propose utilizing the whole scales to help differentiate depressive from negative syndromes.

\subsection{Limitations}

Our psychiatric samples exhibited only mild to moderate negative and depressive symptoms respectively; a greater severity 
of symptoms might have shown the differences between the groups more clearly. Moreover, comparisons between SCZ and MDE groups are difficult since e.g. age of onset and gender ratio differ. However, a matching procedure would compromise representativity either of the schizophrenia or MDE sample. Furthermore, medication could be a confounding variable. Of the subjects with schizophrenia, 95\% were on antipsychotic medication, $19 \%$ took at least one antidepressant. In the MDE group $31 \%$ of the subjects were on antipsychotics (usually in lower doses than the SCZ group), 91\% on antidepressants. Medication-induced blunting could adversely affect the experience of pleasure in subjects with schizophrenia. These secondary negative symptoms could not be ruled out in the present study. Also, our raters were not blinded concerning the subject's diagnosis - this could lead to over- or under-assessement of depressive and/or negative symptoms in concordance with diagnosis.

\subsection{Conclusion}

To differentiate negative symptoms and depression, clinicians might look for self-reported depressive symptoms and observable reduction in expression. The self-report of depressive symptoms is reliable and economical, but more importantly, there is evidence that the self-report might be more sensitive than rater-assessed depressive symptoms in schizophrenia patients [see also 22]. Reduced expression and moderate levels of depression point towards a negative syndrome, whereas relatively unimpaired expression and high scores of self-reported depressive symptoms are more likely to indicate a depressive syndrome.

\section{Funding sources}

This research did not receive any specific grant from funding agencies in the public, commercial, or not-for-profit sectors.

\section{Conflict of interest statement}

On behalf of all authors, the corresponding author states that there is no conflict of interest.

\section{Acknowledgement}

We thank Stefanie Ronge for her help in data acquisition and entry.

\section{References}

[1] Marder S.R., Galderisi S. The current conceptualization of negative symptom in schizophrenia. World Psychiatry 2017:16:14-24.

[2] Kirschner M, Aleman A, Kaiser S. Secondary negative symptoms - A review of mechanisms, assessment and treatment. Schizophr Res 2017;186:29-38.

[3] Upthegrove R, Marwaha S, Birchwood M. Depression and schizophrenia: cause, consequence, or trans-diagnostic issue? Schizophr Bull 2017:43:240-4.

[4] Upthegrove R, Birchwood M, Ross K, Brunett K, McCollum R, Jones L. The evolution of depression and suicidality in first episode psychosis. Acta Psychiatr Scand 2010;122:211-8.

[5] Dutta R, Murray RM, Allardyce J, Jones PB, Boydell J. Early risk factors fo suicide in an epidemiological first episode psychosis cohort. Schizophr Res 2011;126:11-9.

[6] Lako I, Taxis K, Bruggeman R, Knegtering H, Burger H, Wiersma D, et al. The course of depressive symptoms and prescribing patterns of antidepressants in schizophrenia in a one-year follow-up study. Eur Psychiatry 2012;27:240-4.

[7] Fusar-Poli P, Papanastasiou E, Stahl D, Rocchetti M, Carpenter W, Shergill S, et al. Treatments of negative symptoms in schizophrenia: meta-analysis of 168 randomized placebo-controlled trials. Schizophr Bull 2015;41:892-9.

[8] Berenbaum H, Oltmanns TF. Emotional experience and expression in schizophrenia and depression. J Abnorm Psychol 1992;101:37-44.

[9] Tremeau F, Malaspina D, Duval F, Correa H, Hager-Budny M, et al. Facial expressiveness in patients with schizophrenia compared to depressed patients and nonpatient comparison subjects. Am J Psychiatry 2005;162:92-101.

[10] Gaebel W, Wolwer W. Facial expressivity in the course of schizophrenia and depression. Eur Arch Psychiatry Clin Neurosci 2004;254:335-42.
[11] Riehle M, Mehl S, Lincoln TM. The specific social costs of expressive negative symptoms in schizophrenia: reduced smiling predicts interactional outcome. Acta Psychiatr Scand 2018.

[12] Kring AM, Moran EK. Emotional response deficits in schizophrenia: insights from affective science. Schizophr Bull 2008;34:819-34.

[13] Lambert C, Da Silva S, Ceniti AK, Rizvi SJ, Foussias G, Kennedy SH. Anhedonia in depression and schizophrenia: A transdiagnostic challenge. CNS Neurosci Ther 2018.

[14] Gard DE, Kring AM, Gard MG, Horan WP, Green MF. Anhedonia in schizophrenia: Distinctions between anticipatory and consummatory pleasure. Schizophr Res 2007;93:253-60.

[15] Wu H, Mata J, Furman DJ, Whitmer AJ, Gotlib IH, Thompson RJ. Anticipatory and consummatory pleasure and displeasure in major depressive disorder: An experience sampling study. J Abnorm Psychol 2017;126:149-59.

[16] Foussias G, Remington G. Negative symptoms in schizophrenia: avolition and Occam's razor. Schizophr Bull 2010:36:359-69.

[17] Bottlender R, Sato T, Groll C, Jager M, Kunze I, Moller HJ. Negative symptoms in depressed and schizophrenic patients: how do they differ? J Clin Psychiatry 2003;64:954-8.

[18] Park SG, Llerena K, McCarthy JM, Couture SM, Bennett ME, Blanchard JJ. Screening for negative symptoms: preliminary results from the self-report version of the Clinical Assessment Interview for Negative Symptoms. Schizophr Res 2012;135:139-43.

[19] Kring AM, Gur RE, Blanchard JJ, Horan WP, Reise SP. The clinical assessment interview for negative symptoms (CAINS): final development and validation. Am J Psychiatry 2013;170:165-72.

[20] Engel M, Fritzsche A, Lincoln TM. Validation of the german version of the clinical assessment interview for negative symptoms (CAINS). Psychiatry Res 2014;220:659-63.

[21] Llerena K, Park SG, McCarthy JM, Couture SM, Bennett ME, Blanchard JJ. The Motivation and Pleasure Scale-Self-Report (MAP-SR): reliability and validity of a self-report measure of negative symptoms. Compr Psychiatry 2013;54:568-74.

[22] Hartmann MM, Fritzsche A, Lincoln TM. The extent and origin of discordance between self- and observer-rated depression in patients with psychosis. Psychiatry Res 2013;205:247-52.

[23] Engel M, Lincoln TM. Motivation and Pleasure Scale-Self-Report (MAP-SR): validation of the German version of a self-report measure for screening negative symptoms in schizophrenia. Compr Psychiatry 2016;65:110-5.

[24] Hautzinger M. Das Beck-Depressioninventar (BDI) in der Klinik. [The German version of the Beck Depression Inventory (BDI) in clinical use.]. Nervenarzt 1991;62:689-96.

[25] Hamilton M. Development of a rating scale for primary depressive illness. Br J Soc Clin Psychol 1967;6:278-96.

[26] Beck AT, Steer RA, Carbin MG. Psychometric properties of the Beck Depression Inventory: twenty-five years of evaluation. Clin Psychol Rev 1988;8:77-100.

[27] Bagby RM, Ryder AG, Schuller DR, Marshall MB. The Hamilton Depression Rating Scale: Has the Gold Standard Become a Lead Weight? Am J Psychiatry 2004;161:2163-77.

[28] Maier W, Philipp M. Comparative analysis of observer depression scales. Acta Psychiatr Scand 1985;72:239-45.

[29] Gaebel W, Wolwer W. Facial expression and emotional face recognition in schizophrenia and depression. Eur Arch Psychiatry Clin Neurosci 1992;242:46-52.

[30] Browne S, Clarke M, Gervin M, Waddington JL, Larkin C, O'Callaghan E. Determinants of quality of life at first presentation with schizophrenia. Br J Psychiatry 2000;176:173-6.

[31] Katschnig H. Schizophrenia and quality of life. Acta Psychiatr Scand 2000;102:33-7.

[32] Silberstein JM, Pinkham AE, Penn DL, Harvey PD. Self-assessment of social cognitive ability in schizophrenia: association with social cognitive test performance, informant assessments of social cognitive ability, and everyday outcomes. Schizophr Res 2018.

[33] Leucht S, Burkard T, Henderson J, Maj M, Sartorius N. Physical illness and schizophrenia: a review of the literature. Acta Psychiatr Scand 2007:116:317-33.

[34] Greco T, Eckert G, Kroenke K. The outcome of physical symptoms with treatment of depression. J Gen Intern Med 2004;19:813-8.

[35] Baggaley M. Sexual dysfunction in schizophrenia: focus on recent evidence. Hum Psychopharmacol Clin Exp 2008;23:201-9.

[36] Cohrs S. Sleep disturbances in patients with schizophrenia. CNS Drugs 2008;22:939-62.

Janina Richter is a clinical psychologist at the Department of Psychiatry and Psychotherapy, University of Tuebingen. Her main research interest is psychosis with a focus on negative symptoms.

Lea Hölz is a clinical psychologist at the Department of Psychiatry and Psychotherapy, University of Tuebingen. For her Master's thesis she analyzed anhedonia in patients with negative and depressive symptoms.

Klaus Hesse is a clinical psychologist at the Department of Psychiatry and Psychotherapy, University of Tuebingen. His research focus is cognitive behavior therapy for patients with psychosis, in particular relative involvement and improving negative symptoms. 
Dirk Wildgruber is Associate Professor at the Department of Psychiatry and Psychotherapy, University of Tuebingen. His research is mainly focused on social interaction and emotion processing, including the evaluation of specific differences between healthy subjects and patients with psychiatric disorders at the behavioral as well as the neurobiological level.
Stefan Klingberg is Associate Professor of Clinical Psychology and Psychotherapy at the Department of Psychiatry and Psychotherapy, University of Tuebingen. He focuses on psychotherapy in severe mental illness. Major topics of his research are cognitive behavioral therapy for psychosis (CBTp), mechanism of action of CBTp, clinical trials on CBTp, methods of psychotherapy trials, psychopathology of psychosis, assessments of psychosis. 